\title{
Amplitude Modulation in a Molecular Communication Testbed with Superparamagnetic Iron Oxide Nanoparticles and a Micropump
}

This paper was downloaded from TechRxiv (https://www.techrxiv.org).

\section{LICENSE}

CC BY-NC-SA 4.0

SUBMISSION DATE / POSTED DATE

$11-01-2021 / 13-01-2021$

\section{CITATION}

Bartunik, Max; Thalhofer, Thomas; Wald, Christian; Richter, Martin; Fischer, Georg; Kirchner, Jens (2021): Amplitude Modulation in a Molecular Communication Testbed with Superparamagnetic Iron Oxide Nanoparticles and a Micropump. TechRxiv. Preprint. https://doi.org/10.36227/techrxiv.13554458.v1

$\mathrm{DOI}$ 


\title{
Amplitude Modulation in a Molecular Communication Testbed with Superparamagnetic Iron Oxide Nanoparticles and a Micropump
}

\author{
Max Bartunik ${ }^{1[0000-0002-3033-5798]}$ Thomas Thalhofer ${ }^{2}$, Christian Wald ${ }^{2}$, \\ Martin Richter ${ }^{2}$, Georg Fischer ${ }^{10000-0002-4033-2005]}$, and Jens \\ Kirchner ${ }^{10000-0002-8623-9551]}$ \\ 1 Institute for Electronis Engineering, Friedrich-Alexander-Universität \\ Erlangen-Nürnberg (FAU), Germany \\ max.bartunik@fau.de \\ 2 Fraunhofer EMFT Research Institution for Microsystems and Solid State \\ Technologies, Munich, Germany
}

\begin{abstract}
Molecular communication uses molecules or other nanoscale particles to transmit data in scenarios where conventional communication techniques are not feasible. In previous work a testbed using superparamagnetic iron oxide nanoparticles (SPIONs) as information carriers in a fluid transmission channel with constant background flow was proposed. The SPIONs are detected at a receiver as change of a coils inductance. We now improve the testbed by using a piezoelectric micropump as transmitter, making amplitude modulation (AM) with different injection volumes possible. Machine learning is employed at the receiver to differentiate between six different amplitude levels and grey code is used to reduce bit errors. With AM and the designed coding scheme, the achievable effective data rate was doubled to $4.45 \mathrm{bit} \mathrm{s}^{-1}$.
\end{abstract}

\section{Introduction}

The aim of molecular communication is to achieve data transmission using molecules or other particles in the nanoscale. So far, setups to experimentally investigate molecular communication with various transmission methods, such as air-based transmission with alcohol $[3,8,18]$ or encoding information as change of $\mathrm{pH}$-value $[4,6,9]$ have been proposed. In [16] a different setup that uses superparamagnetic iron oxide nanoparticles (SPIONs) as information carriers in a fluid transmission channel is presented. $[1,2]$ provide some induction-based receiver concepts for such SPION-based testbeds.

Investigated data transmission scenarios in $[1,2]$ were confined to binarystate symbol transmissions, encoding a ' 1 ' as an injection of SPIONs into the transmission channel and a ' 0 ' as no injection. The achievable information rate was restricted due to an increase of inter-symbol interference for reduced symbol intervals. 
Schemes for amplitude modulation (AM), or concentration shift keying, in molecular communication have been proposed previously (e.g. $[10,14])$. However, these focus on a diffusion-based scenario in contrast to the active transport present in the provided testbed and are restricted to theoretical channel observations. In $[2,16]$ a peristaltic pump was used as a transmitter to enable on-off keying (OOK). However, the pump was limited to a fixed transmission volume. We now replace the peristaltic pump with a micropump allowing for use of AM to achieve a higher-level encoding per symbol. Machine learning is used at the receiver to detect the transmitted amplitude levels.

Today, micropumps are used in many applications including drug delivery [5], lab-on-a-chip devices [13] and sensor enhancement [12]. As described in [15, 19] many different actuators have been used for micropumps. Of these technologies, piezoelectric actuated micropumps show the most promising features since they are able to create very high forces and actuation speeds, enabling high pumping frequencies and pressures. For this paper a refined version of the steel-foil based micropump introduced in [17] with a diameter of $20 \mathrm{~mm}$, designed by Fraunhofer Research Institution for Microsystems and Solid State Technologies (EMFT) in Munich, was used. The pumps parameters allow a fast injection of a well-defined, discrete volume of SPIONs of up to $11.5 \mu \mathrm{l}$ per pump stroke. The stroke volume can be controlled by changing the piezo actuator voltage enabling a variety of amplitude levels.

We used the described micropump to achieve AM in a molecular communication testbed with SPIONs for the first time. Together with a comprehensive coding scheme, employing two-dimensional grey code and symbol detection based on machine learning, the achievable data rate was doubled in comparison to previous setups using OOK.

In the following, we will first describe the testbed setup and its individual components. Then we will present the methods used to encode and decode data, as well as the achieved data transmission in the experimental testbed. A discussion of the results and an outline of future improvements concludes the article.

\section{Testbed}

The testbed can be separated into the four hardware components information carriers, transmitter, channel and receiver. The principle setup was first described in [16].

\subsection{Information Carriers}

Information is transmitted in the testbed by changing the concentration of SPIONs. Due to their superparamagnetic properties they can be detected as change of a coils inductance. In our case we used particles with a hydrodynamic diameter of $50 \mathrm{~nm}$, a specific susceptibility of $8.78 \times 10^{-3}$ for an aqueous solution with a concentration of $1 \mathrm{mg} \mathrm{Fe} \mathrm{ml}^{-1}$ and a coating of lauric acid. They were synthesized by the Section for Experimental Oncology and Nanomedicine (SEON) of the University Hospital Erlangen. 


\subsection{Transmission Channel}

A flexible tube with an inner diameter of $1.52 \mathrm{~mm}$ is used as the transmission channel. A constant background flow of water is provided by a peristaltic pump (Ismatec ISM596D) with a flow rate of $10 \mathrm{ml} \mathrm{min}^{-1}$. SPIONs are injected into the transmission tube as described in section 2.3 and travel a distance of $5 \mathrm{~cm}$ to the receiver. Due to laminar flow the injected nanoparticles are spread out axially along the transmission channel, causing inter-symbol interference for repeated injections.

\subsection{Transmitter}

The transmitter consists of a steel micropump, manufactured by Fraunhofer EMFT in Munich, and a venous cannula.

The pump has a height of $1 \mathrm{~mm}$, a diameter of $20 \mathrm{~mm}$ and moves a volume of $11 \mathrm{\mu l}$ per full stroke. Due to its high flow rate of up to $20 \mathrm{ml} \mathrm{min}^{-1}$ (for water), and high pressure tolerance of $60 \mathrm{kPa}$, it is ideal for use in the testbed environment. As can be seen in Fig. 1 and 2, the micropump is composed of a coin shaped steel base plate, three steel foils and a piezo actuator disc. Two steel foils are laser-welded on top of the base plate to create passive inlet- and outlet valves and valve seats. The third steel foil closes the top of the pump chamber. The piezo actuator is glued onto the pump chamber with a patented process described in $[7]$.

The piezo actuator is driven with a rectangular voltage signal. The stroke volume can be adjusted by varying the voltage amplitude: A signal alternating from $-80 \mathrm{~V}$ to $+300 \mathrm{~V}$ results in a full stroke with an approximate volume of $11 \mu \mathrm{l}$. For an input signal with a reduced voltage swing of $-80 \mathrm{~V}$ to $+150 \mathrm{~V}$ a volume of approximately $6 \mathrm{\mu l}$ is expedited per stroke.

A venous cannula with an inner diameter of $0.8 \mathrm{~mm}$ is used to inject the nanoparticles into the transmission channel. The cannula is placed so that the injection occurs in the middle of the channel in the same direction as the background flow.

The amount of SPIONs that are introduced into the background channel in each symbol interval is varied to achieve different amplitude levels. As the micropump is operated at a significantly higher rate $(40 \mathrm{~Hz})$ than the symbol frequency (maximally $3.5 \mathrm{~Hz}$ ), multiple pump strokes are observed as an individual injection with higher volume. Furthermore, the injected volume per pump stroke can be controlled. We therefore chose six different amplitude levels with steps of a half stroke (approx. $6 \mathrm{\mu l}$ ) ranging from no injection $(0 \mathrm{\mu l})$ to two and a half strokes (approx. $28 \mathrm{\mu l}$ ) per injection.

\subsection{Receiver}

The transmission channel is passed through a custom made coil at the receiver. Due to the magnetic properties of the SPIONs the coil inductance changes as the nanoparticles pass through it. In a parallel circuit this change of inductance can 


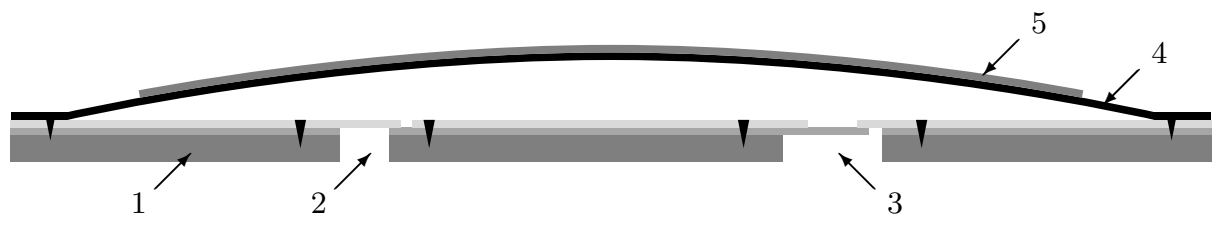

(a) The pump consists of a steel base plate (1), passive inlet (2) and outlet (3) valves, an actuator diaphragm (4) and the piezoelectric actuator (5). Here the initial state, when no voltage is applied to the actuator, is shown. The mounting process leads to a pre-bent piezo actuator and actuator diaphragm.

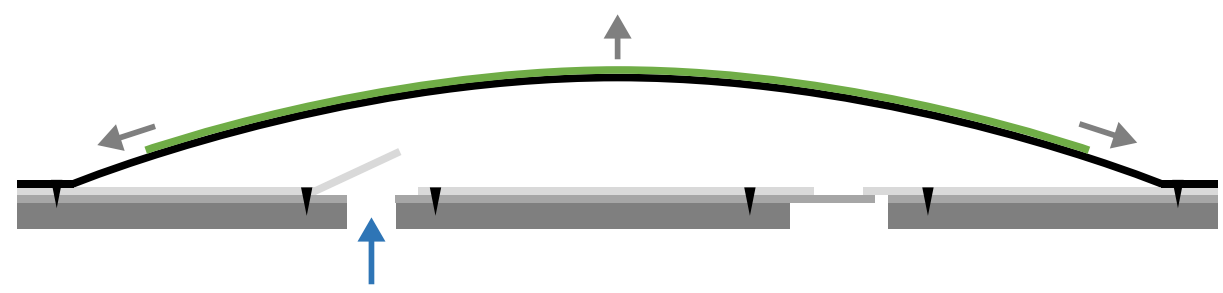

(b) Applying a negative voltage of $-80 \mathrm{~V}$ to the actuator causes the diaphragm to bend upwards and the pump chamber is filled.

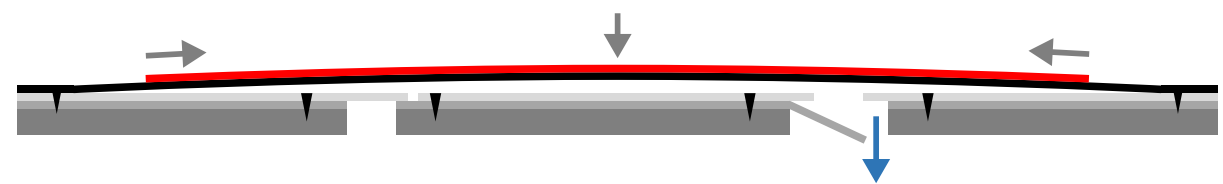

(c) With a positive voltage in the range of $80 \mathrm{~V}$ to $300 \mathrm{~V}$ the diaphragm is bent downwards and the liquid in the pump chamber is ejected through the outlet valve.

Fig. 1. Schematic cross-section of the used steel micropump shown for the three different actuation states. 


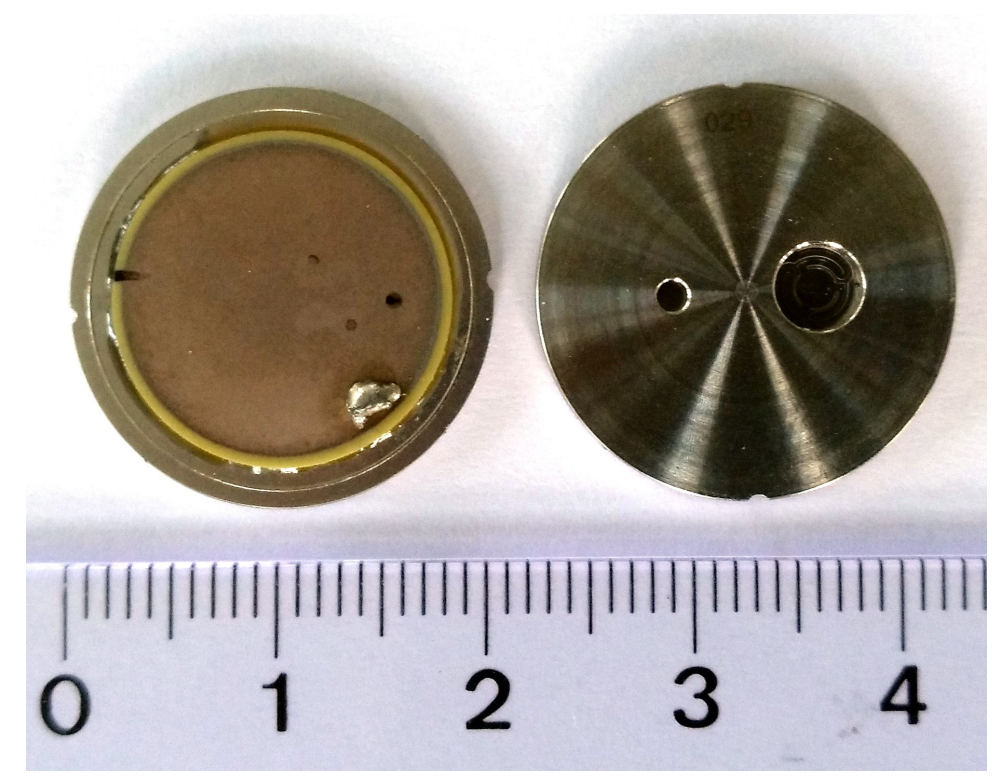

Fig. 2. Pictures of the steel micropump showing the top with the piezoelectric actuator (left) and the steel base plate (right). The pump has a diameter of $20 \mathrm{~mm}$.

be observed as a shift of resonance frequency that is measured using the LDC1612 from Texas Instruments. The receiver setup and capabilities were presented in [2]. The change in resonance frequency depends on the amount of SPIONs inside the detection coil, allowing for differentiation of various amplitude levels.

\section{Data Transmission}

Data transmission in the testbed is achieved by encoding binary data in six different amplitude levels (as described in section 2.3) that are detected at the receiver and decoded using machine learning classification.

\subsection{Encoding}

Each transmitted symbol has six possible amplitude levels (0 to 5). Words are constructed of two symbols with one amplitude level each. Binary data is represented by encoding five bits to one word. When encoding all possible 32 combinations of five bits to two symbol words (36 possible combinations), 4 unused words remain. In future these may be used for transmission synchronisation or other control commands. In our case these remaining code words are used to increase the distance between similar amplitude levels. Furthermore, to reduce bit errors in scenarios where a detected amplitude level is incorrect but close to the actually transmitted level, a two-dimensional grey code is applied, changing only one bit for neighbouring words. Table 1 shows the complete encoding. 
Table 1. Encoding table for the representation of 5 bits using words consisting of two symbols $\left(\mathrm{S}_{0}\right.$ and $\left.\mathrm{S}_{1}\right)$. To reduce bit errors for similar amplitude levels neighbouring words have only a single bit change. The four remaining words in blue are not used for transmission. A neighbouring bit sequence was arbitrarily chosen for decoding.

\begin{tabular}{lcccccc}
\hline & \multicolumn{6}{c}{$\mathbf{S}_{\mathbf{1}}$} \\
\cline { 2 - 7 } $\mathbf{S}_{\mathbf{0}}$ & 0 & 1 & 2 & 3 & 4 & 5 \\
\hline 0 & 00000 & 00001 & 00011 & 01011 & 01001 & 01000 \\
1 & 00100 & 00001 & 00010 & 01010 & 01001 & 01100 \\
2 & 00101 & 00111 & 00110 & 01110 & 01111 & 01101 \\
3 & 10101 & 10111 & 10110 & 11110 & 11111 & 11101 \\
4 & 10100 & 10001 & 10010 & 11010 & 11001 & 11100 \\
5 & 10000 & 10001 & 10011 & 11011 & 11001 & 11000 \\
\hline
\end{tabular}

To enable simple detection, each transmission is initiated and terminated with a symbol of maximal amplitude (value 5).

\subsection{Decoding}

The recorded signal is decoded using machine learning in a Matlab (The MathWorks, Inc.) script. First, a moving averaging filter with a length of two samples is applied to reduce noise and the amount of data points is increased to $100 \mathrm{Sa} \mathrm{s}^{-1}$ with cubic spline interpolation. Next, a zero baseline is determined as the mean value of two symbol intervals before injection of SPIONs. The initial synchronisation peak is set to the first local maximum after a threshold at half of the maximal signal amplitude is exceeded. This peak is used to determine the fixed symbol intervals with a known duration. To accommodate for transmission timing inaccuracies, the symbol intervals are resynchronised at the highest available peaks that are at least five symbol intervals apart and exceed the initial detection threshold.

For each symbol interval three signal parameters, which were determined by a feature selection algorithm, are calculated. First, local maxima inside the symbol interval are searched. For the peak with the largest amplitude the height of the rising and falling edges are calculated by determining the minimal amplitude within one symbol interval before and after the peak. In addition, the absolute amplitude value is also recorded. If no local maximum is found, the symbol amplitude is set to the minimal amplitude inside the symbol interval. Fig. 3 shows a section with eight symbols of a sample transmission, demonstrating detection of the prediction parameters. As inter-symbol interference causes a changed baseline for each symbol interval, the application of fixed threshold values to differentiate between multiple amplitude levels is not possible.

The rising and falling edge heights are normalised to the values of the initial synchronisation peak and then passed to a classification predictor together with the amplitude levels. A discriminant analysis model fitted to a training sample sequence with a total of 204 symbols is used for classification. An individual 


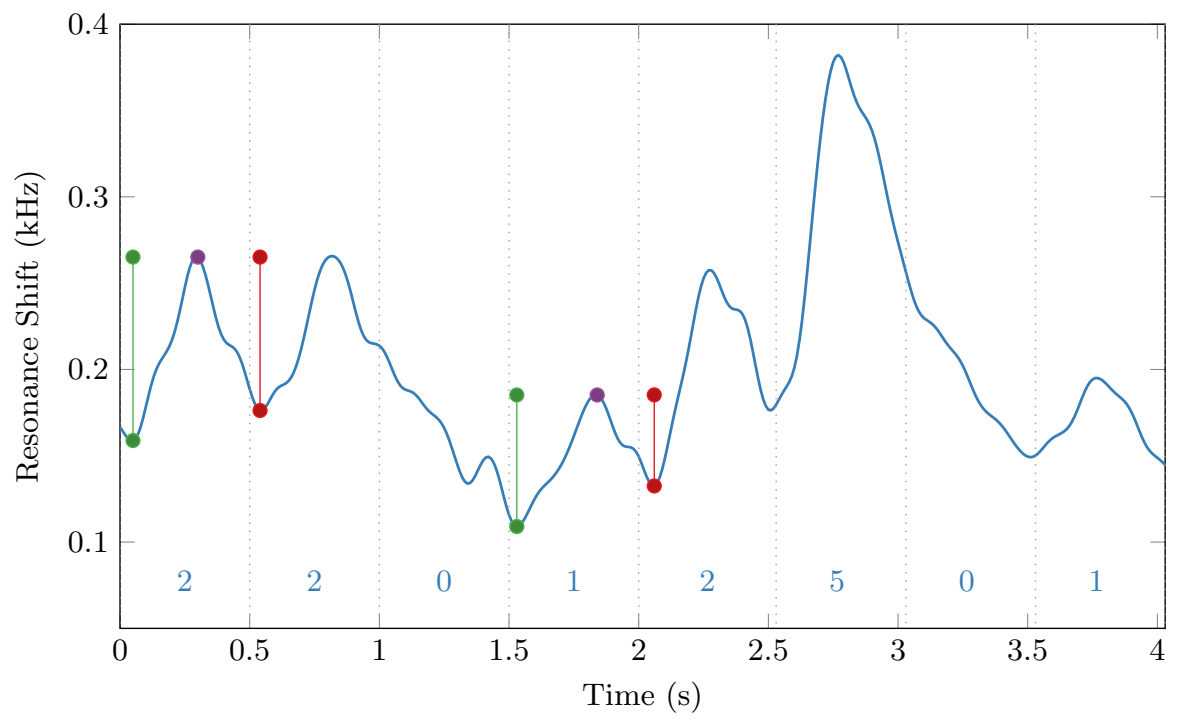

Fig. 3. Sample transmission sequence section with eight symbols. The symbol intervals are shown with dashed lines and are annotated with the transmitted amplitude level. Peaks are detected and the parameters peak amplitude (purple), rising edge height (green) and falling edge height (red) determined for each symbol interval. The parameter detection is shown exemplary for two symbols. 
model was trained for each symbol rate $(2 \mathrm{~Hz}$ to $3.5 \mathrm{~Hz})$ with the 'fit discriminant analysis classifier' algorithm in Matlab. Fig. 4 shows the spread of the three prediction parameters in the training sequence for a symbol rate of $2 \mathrm{~Hz}$. The high deviation of the predictor parameters with overlapping areas does not allow for simple threshold detection. By using the combined information of all three parameters in a machine learning model a good prediction of the transmitted symbol can be made.

\section{Results}

\subsection{Injection Volume Correlation}

In a first step, the correlation between injected volume of SPIONs and detected shift in resonance frequency is of interest. To determine this relation a series of injections were performed with the micropump ranging in volume from one to five pump strokes. Between each injection a pause, sufficient to ensure a return to zero, was made. Fig. 5 shows the detected resonance shift, normalised to the maximal value, in relation to the normalised injection volume. As expected, a linear relation between the detection signal and the injection volume can be observed. Therefore, detection of different amplitude levels is possible.

\subsection{Data Transmission}

Information en- and decoding was evaluated for four different symbol rates ranging from $2 \mathrm{~Hz}$ to $3.5 \mathrm{~Hz}$ in steps of $0.5 \mathrm{~Hz}$, each with a random sequence of 2000 bits. Due to restrictions for the micropump driver the sequence was split into four transmissions of 100 symbols. Each sequence was decoded as described in section 3.2 and the binary output evaluated for bit errors.

Fig. 6 shows a sample transmission section consisting of eight symbols. All words (i. e. symbol tuples) but one were decoded correctly. Due to the choice of coding only a single bit error results from the incorrectly detected word.

In Fig. 7 the average symbol and bit error probabilities are shown for the four different symbol interval durations. No significant correlation between reduced symbol intervals and an increase of decoding errors can be observed. As can be seen in Fig. 8, symbol detection errors are restricted to a single value offset in most cases. Due to the choice of coding, only a single bit error occurs for such a single value detection offset. The result is a significantly lower bit error rate ( $20 \%$ for a single word with a typical symbol error) than symbol error rate $(50 \%$ for a single word with one detection error).

From an information theory perspective our transmission from encoding to decoding can be regarded as a binary symmetric channel, implying equal probabilities for bit errors independent of bit value. The capacity of such a channel with a given error probability $f$ is

$$
C(f)=1-\left(f \log _{2} \frac{1}{f}+(1-f) \log _{2} \frac{1}{1-f}\right) .
$$



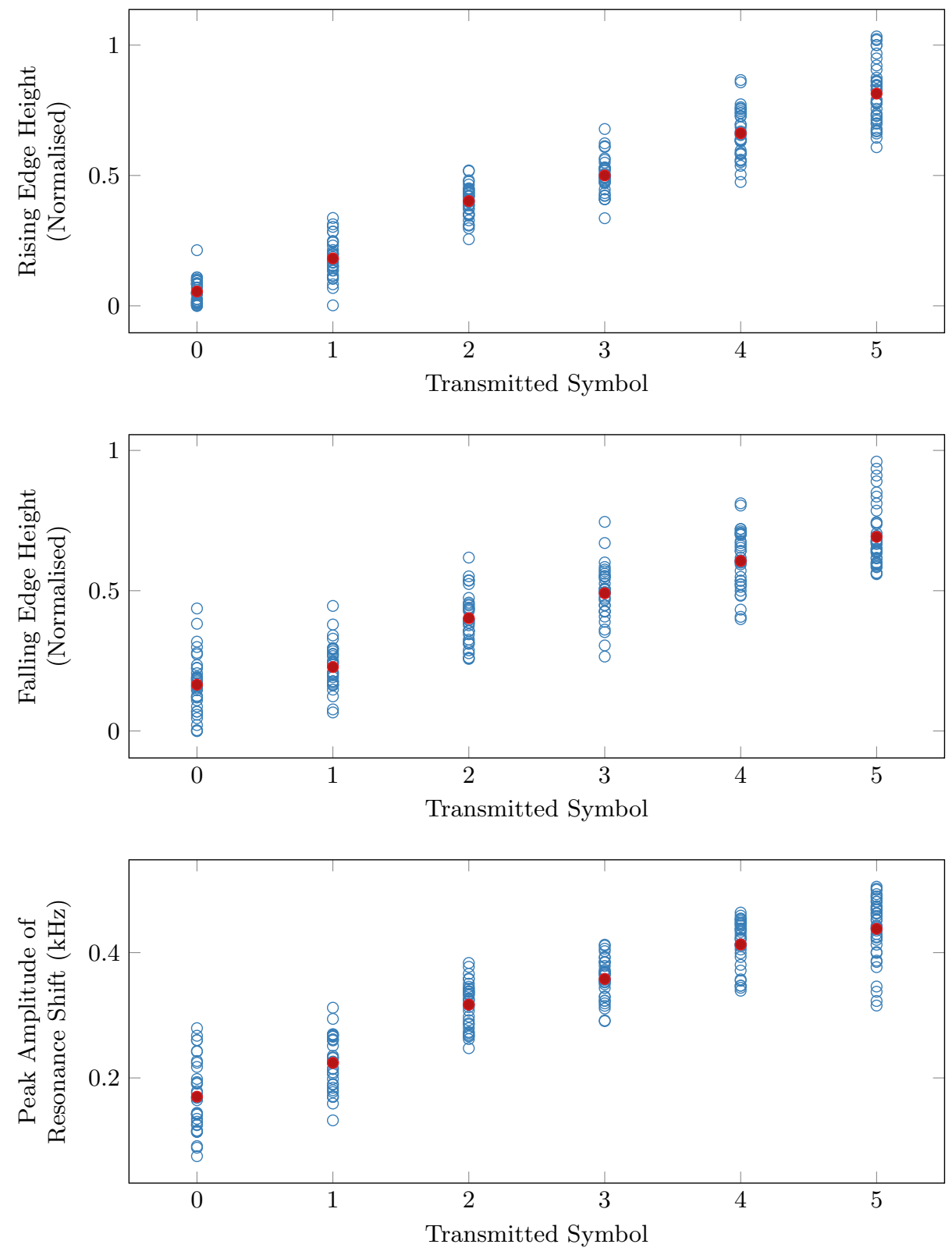

Fig. 4. Distribution of the three predictor parameters rising edge height, falling edge height and peak amplitude for a training set of 204 symbols. Although the mean values marked in red show a clear tendency, definite detection thresholds cannot be set due to the high deviations. 


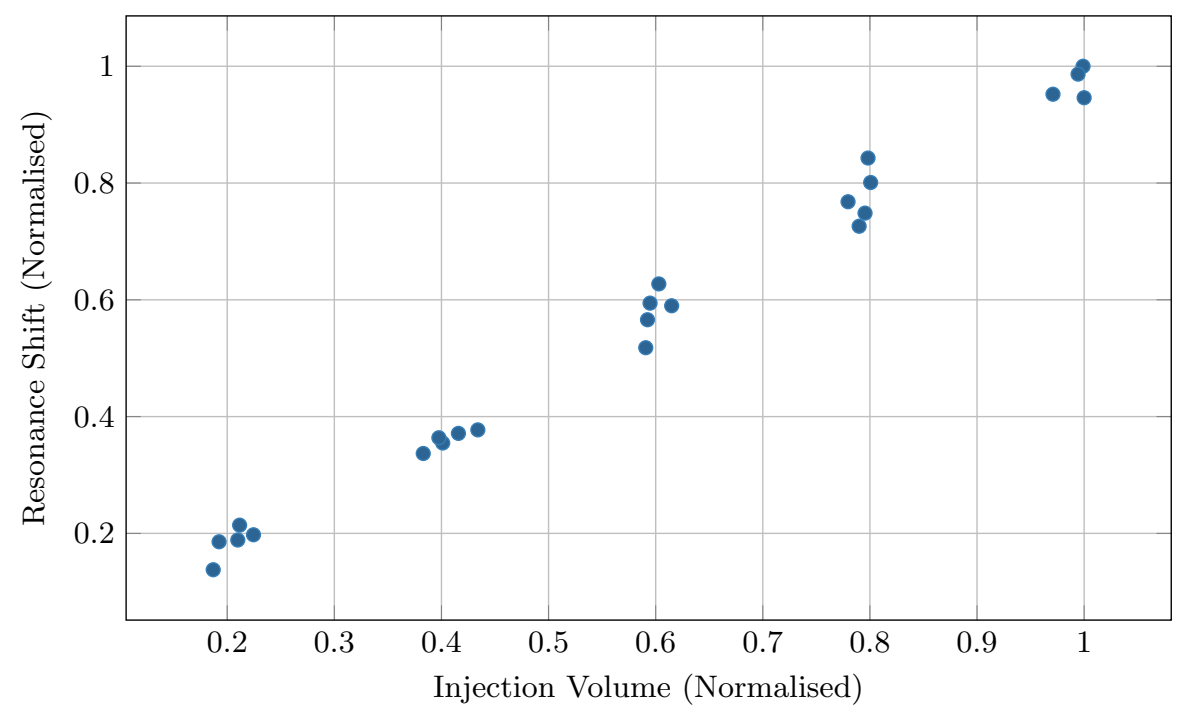

Fig. 5. Resonance shift at the receiver in relation to injection volume for 24 individual injection bursts. Each burst consists of one to five pump strokes. A linear relation between the received signal and the transmitted volume of SPIONs can be observed.

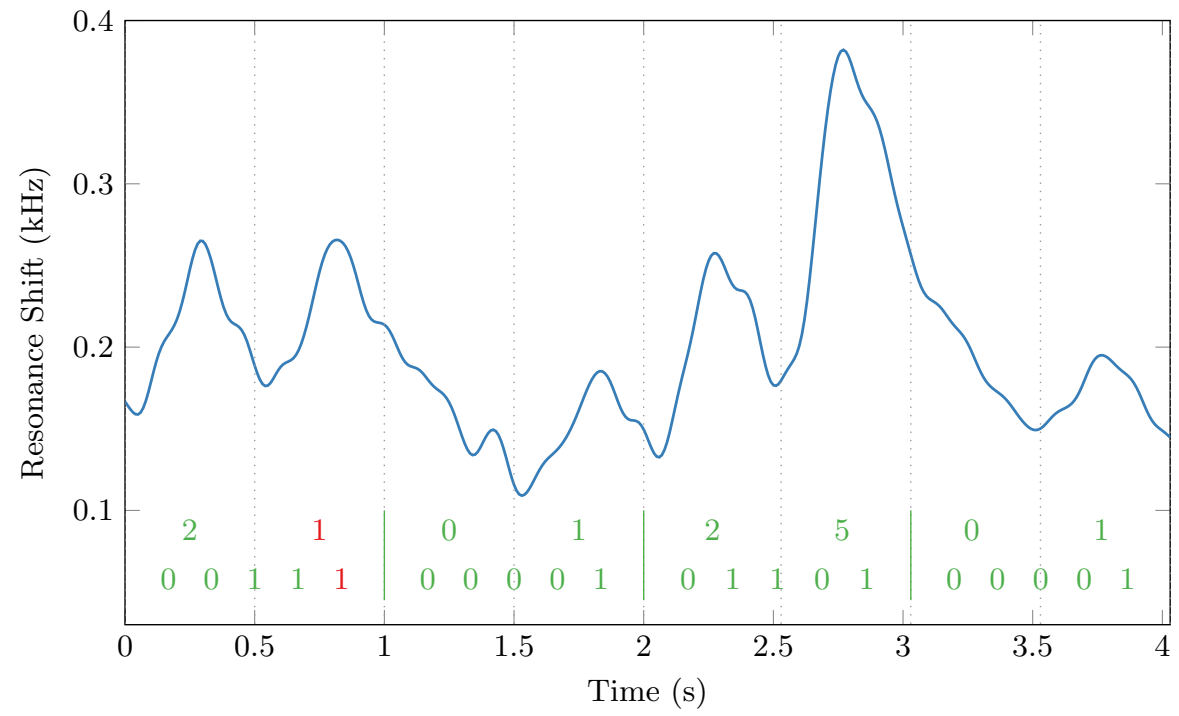

Fig. 6. Sample transmission sequence section with eight symbols. The detected symbol values for each interval and the decoded words are shown. The second transmitted symbol value (2) was incorrectly detected as 1 . However, as neighbouring words differ only by a one bit the selected coding restricts the decoded binary word to a single bit error. 


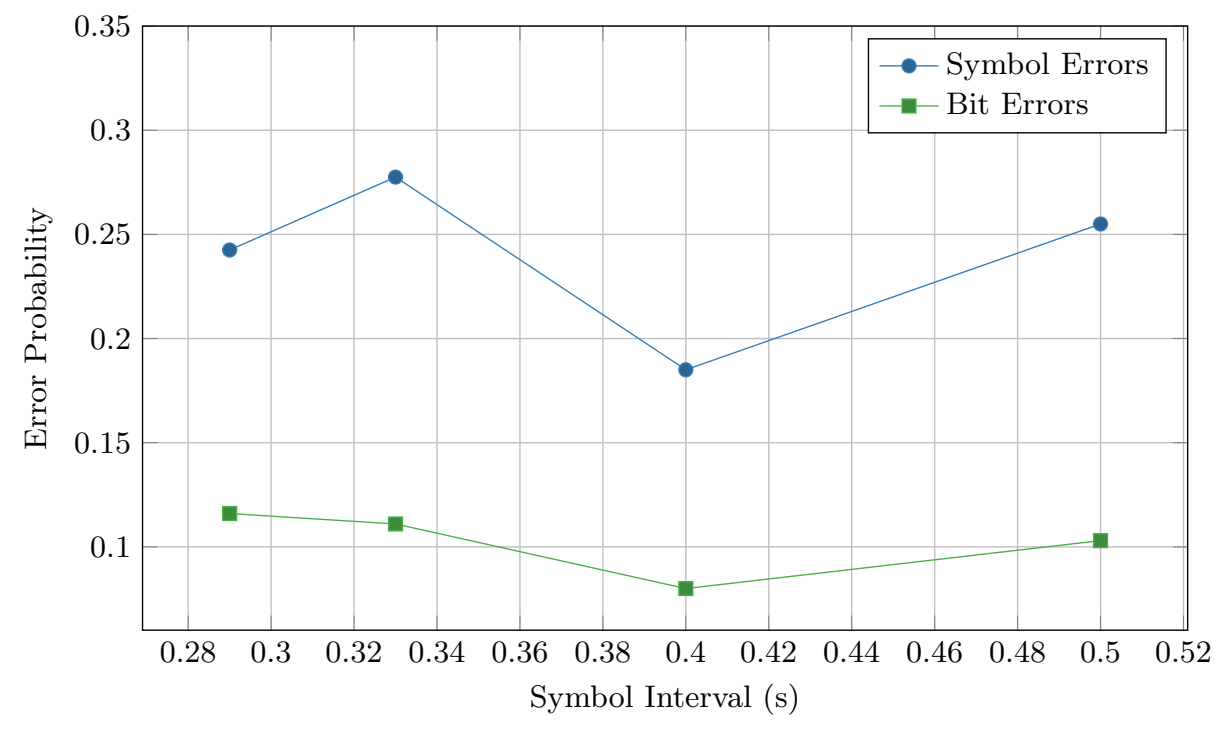

Fig. 7. Probabilities for bit and symbol errors using amplitude modulation and the provided coding scheme. Due to the use of grey code, the average probability for a bit error $(10.3 \%)$ is significantly lower than the symbol error probability $(24 \%)$.

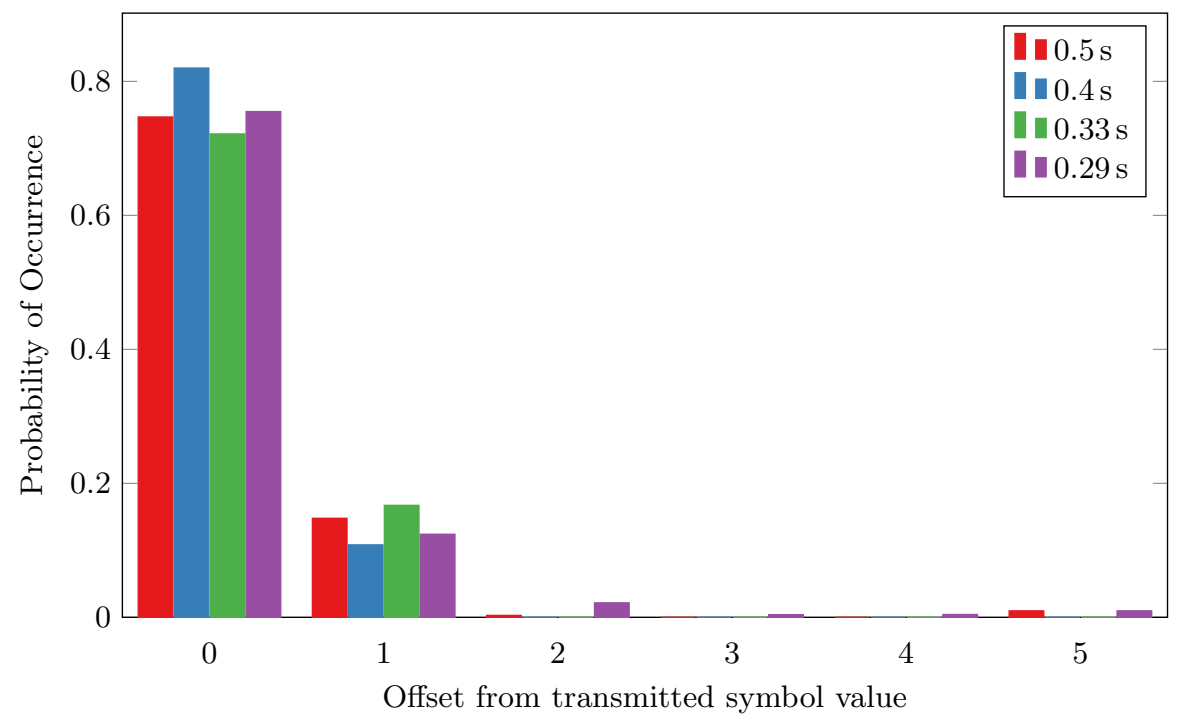

Fig. 8. Probabilities for various receive symbol errors. A detection error with an offset larger than 1 amplitude level occurs in only very few cases, resulting in the coding gain achieved by the use of grey code. 
The noisy-channel coding theorem provides an upper boundary for achievable effective data rate given a channel capacity and the tolerable bit error rate (BER) [11]. This effective data rate is given by

$$
\frac{C}{R}=1-\left(\mathrm{BER}_{\log _{2}} \frac{1}{\mathrm{BER}}+(1-\mathrm{BER}) \log _{2} \frac{1}{1-\mathrm{BER}}\right)
$$

where $R$ is the code rate. Using (2) we can calculate the possible effective data rates for both the transmission scenario using $\mathrm{AM}$ and previous results with OOK presented in [2].

Fig. 9 shows the calculated values for the various symbol intervals and multiple tolerable BER scenarios. Although different symbol intervals were investigated in [2], the possible effective data rates can be compared to the results presented here. For a symbol interval of $0.4 \mathrm{~s}$ and a tolerable BER of $1 \%$ an effective data rate of $3.9 \mathrm{bit} \mathrm{s}^{-1}$ is achievable using AM. With the same parameters the data rate was restricted to $2.3 \mathrm{bits}^{-1}$ using OOK. Of the investigated intervals, the maximal data rate using $\mathrm{AM}$ is reached at a symbol interval of $0.29 \mathrm{~s}$ and is $4.5 \mathrm{bit} \mathrm{s}^{-1}$ for a remaining BER of $1 \%$. This is close to double the value achievable with OOK in [2].

\section{Conclusion}

A micropump was successfully used as a transmitter in the provided molecular communication testbed. Due to the pumps capabilities, six different amplitude levels could be differentiated at the receiver by modulating the volume of SPIONs injected in one symbol interval. With machine learning and an adapted coding a bit error probability of $10.3 \%$ was achieved.

As no significant increase of bit errors for reduced symbol intervals was observed, a further increase of symbol rate and therefore effective data rate is possible and will have to be investigated in future work. Limiting factors may be the increase of inter-symbol interference due to laminar flow and the sensor sample rate.

In comparison to data transmission using on-off keying presented in [2] the achievable effective data rate was doubled.

In future work improvements may be made on both the coding scheme and the testbed setup. In particular, an increase of the sensor sample rate and an optimisation of the used coils is of interest. Symbol encoding could be optimised to ensure larger amplitude changes and therefore reduce bit errors. Finally, the machine learning model used for detecting amplitude values could be improved with a significantly larger training set.

\section{Acknowledgements}

The authors would like to express their sincere gratitude to Harald Unterweger from the Section for Experimental Oncology and Nanomedicine of the University 


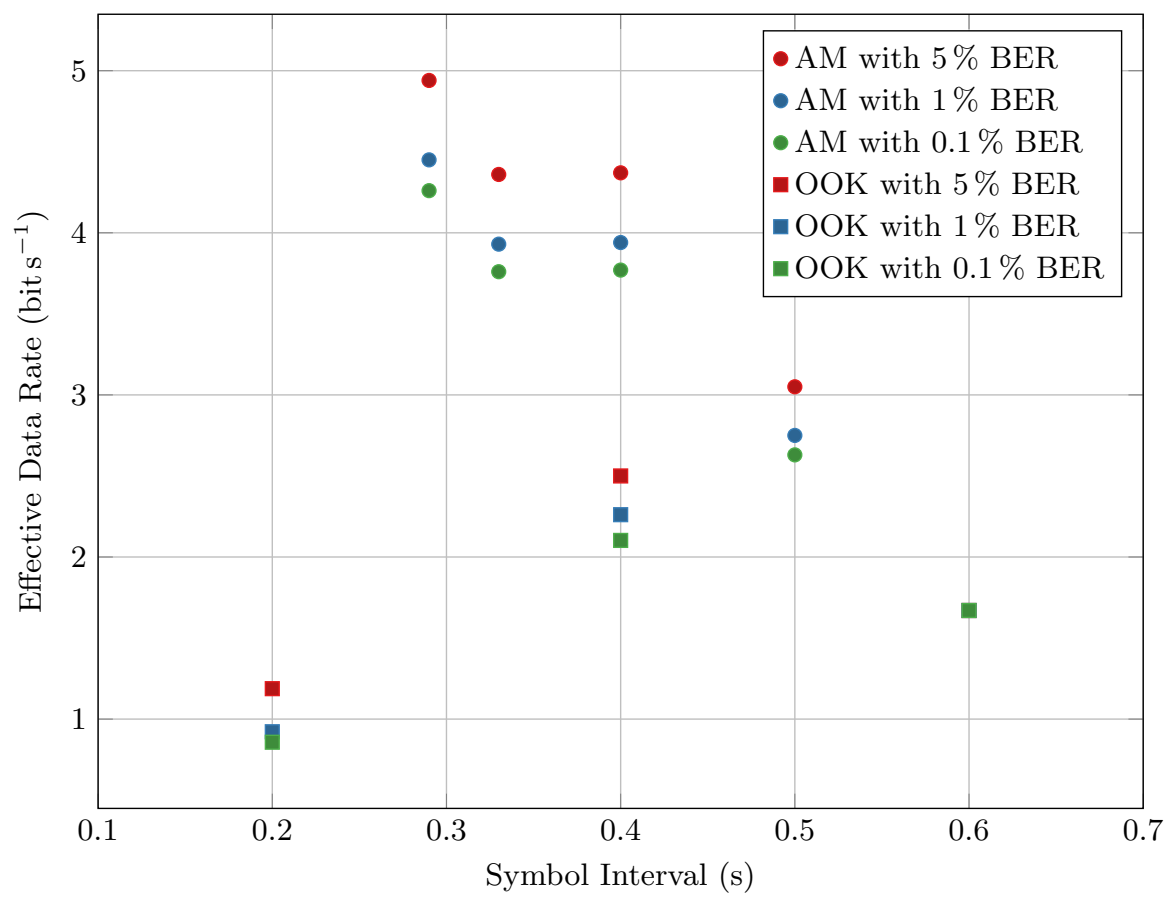

Fig. 9. Achievable effective data rate according to the noisy-channel coding theorem. Comparable values for OOK were taken from [2]. With a tolerable BER of $1 \%$ an effective data rate of $4.5 \mathrm{bits}^{-1}$ can be reached using the proposed AM scheme. The maximal value for $\mathrm{OOK}$ is $2.3 \mathrm{bits}^{-1}$. 
Hospital Erlangen for manufacturing and providing continuous support regarding SPIONs.

This work was supported in part by the German Federal Ministry of Education and Research (BMBF), project MAMOKO (16KIS0913K).

\section{References}

1. Bartunik, M., Lübke, M., Unterweger, H., Alexiou, C., Meyer, S., Ahmed, D., Fischer, G., Wicke, W., Jamali, V., Schober, R., Kirchner, J.: Novel receiver for superparamagnetic iron oxide nanoparticles in a molecular communication setting. In: Proceedings of the Sixth Annual ACM International Conference on Nanoscale Computing and Communication - NANOCOM '19. ACM Press (Sep 2019). https://doi.org/10.1145/3345312.3345483

2. Bartunik, M., Unterweger, H., Alexiou, C., Schober, R., Lübke, M., Fischer, G., Kirchner, J.: Comparative evaluation of a new sensor for superparamagnetic iron oxide nanoparticles in a molecular communication setting. In: Bio-inspired Information and Communication Technologies. pp. 303-316. Springer International Publishing, Cham (2020). https://doi.org/10.1007/978-3-030-57115-3_27

3. Farsad, N., Guo, W., Eckford, A.W.: Tabletop molecular communication: Text messages through chemical signals. PLOS ONE 8(12), 1-13 (Dec 2013). https://doi.org/10.1371/journal.pone.0082935

4. Farsad, N., Pan, D., Goldsmith, A.: A novel experimental platform for in-vessel multi-chemical molecular communications. In: GLOBECOM 2017 - 2017 IEEE Global Communications Conference. IEEE (Dec 2017). https://doi.org/10.1109/glocom.2017.8255058

5. Ginsberg, B.H.: Patch pumps for insulin. Journal of Diabetes Science and Technology 13(1), 27-33 (2019). https://doi.org/10.1177/1932296818786513

6. Grebenstein, L., Kirchner, J., Peixoto, R.S., Zimmermann, W., Irnstorfer, F., Wicke, W., Ahmadzadeh, A., Jamali, V., Fischer, G., Weigel, R., Burkovski, A., Schober, R.: Biological optical-to-chemical signal conversion interface: A smallscale modulator for molecular communications. IEEE Transactions on NanoBioscience 18(1), 31-42 (Jan 2019). https://doi.org/10.1109/tnb.2018.2870910

7. Herz, M., Richter, M., Wackerle, M.: Method for manufacturing a bending transducer, a micro pump and a micro valve, micro pump and micro valve (2016), https://patents.google.com/patent/US9410641B2/en

8. Koo, B.H., Lee, C., Yilmaz, H.B., Farsad, N., Eckford, A., Chae, C.B.: Molecular MIMO: From theory to prototype. IEEE Journal on Selected Areas in Communications 34(3), 600-614 (Mar 2016). https://doi.org/10.1109/jsac.2016.2525538

9. Krishnaswamy, B., Austin, C.M., Bardill, J.P., Russakow, D., Holst, G.L., Hammer, B.K., Forest, C.R., Sivakumar, R.: Time-elapse communication: Bacterial communication on a microfluidic chip. IEEE Transactions on Communications 61(12), 5139-5151 (Dec 2013). https://doi.org/10.1109/tcomm.2013.111013.130314

10. Kuran, M.S., Yilmaz, H.B., Tugcu, T., Akyildiz, I.F.: Modulation techniques for communication via diffusion in nanonetworks. In: 2011 IEEE International Conference on Communications (ICC). pp. 1-5 (Jun 2011)

11. MacKay, D.J.C.: Information Theory, Inference and Learning Algorithms. Cambridge University Press (Oct 2003)

12. Müller, G., Friedberger, A., Kreisl, P., Ahlers, S., Schulz, O., Becker, T.: A mems toolkit for metal-oxide-based gas sensing systems. Thin Solid Films 436(1), 34-45 (Jul 2003). https://doi.org/10.1016/S0040-6090(03)00523-6 
13. Pabst, O., Perelaer, J., Beckert, E., Schubert, U.S., Eberhardt, R., Tünnermann, A.: All inkjet-printed piezoelectric polymer actuators: Characterization and applications for micropumps in lab-on-a-chip systems. Organic Electronics 14(12), 3423 - 3429 (Dec 2013). https://doi.org/10.1016/j.orgel.2013.09.009

14. Singhal, A., Mallik, R.K., Lall, B.: Performance analysis of amplitude modulation schemes for diffusion-based molecular communication. IEEE Transactions on Wireless Communications 14(10), 5681-5691 (Jun 2015). https://doi.org/10.1109/TWC.2015.2441067

15. Tsai, N.C., Sue, C.Y.: Review of mems-based drug delivery and dosing systems. Sensors and Actuators A: Physical 134(2), 555 - 564 (Mar 2007). https://doi.org/10.1016/j.sna.2006.06.014

16. Unterweger, H., Kirchner, J., Wicke, W., Ahmadzadeh, A., Ahmed, D., Jamali, V., Alexiou, C., Fischer, G., Schober, R.: Experimental molecular communication testbed based on magnetic nanoparticles in duct flow. In: 2018 IEEE $19^{\text {th }}$ International Workshop on Signal Processing Advances in Wireless Communications (SPAWC). pp. 1-5 (Jun 2018). https://doi.org/10.1109/SPAWC.2018.8446011

17. Wald, C., Richter, M.: Low-Cost Edelstahl-Mikropumpe zur Probenzufuhr eines miniaturisierten Gassensorsystems zur Brandfrüherkennung. MikroSystemTechnik Kongress Proceedings. Mikroelemente \& Systeme. 2015, 448-451 (Oct 2015)

18. Wang, L., Farsad, N., Guo, W., Magierowski, S., Eckford, A.W.: Molecular barcodes: Information transmission via persistent chemical tags. In: 2015 IEEE International Conference on Communications (ICC). IEEE (Jun 2015). https://doi.org/10.1109/icc.2015.7248469

19. Wang, Y.N., Fu, L.M.: Micropumps and biomedical applications - a review. Microelectronic Engineering 195, 121 - 138 (2018). https://doi.org/10.1016/j.mee.2018.04.008 